\title{
ON THE INVERSE CASCADE OF MAGNETIC HELICITY
}

\author{
Alexandros Alexakis, ${ }^{1}$ Pablo D. Mininni, ${ }^{1}$ and Annick Pouquet ${ }^{1}$ \\ Received 2005 September 15; accepted 2005 November 18
}

\begin{abstract}
We study the inverse cascade of magnetic helicity in conducting fluids, as pertinent to the generation and dynamics of magnetic fields as observed, e.g., in the solar corona, by investigating the detailed transfer of helicity between different spherical shells in Fourier space in direct numerical simulations of three-dimensional magnetohydrodynamics (MHD). Two different numerical simulations are used, one in which the system is forced with an electromotive force in the induction equation and one in which the system is forced mechanically with an $\mathrm{ABC}$ flow and the magnetic field is solely sustained by a dynamo action. The magnetic helicity cascade at the initial stages of both simulations is observed to be inverse and local (in scale space) at large scales and direct and local at small scales. When saturation is approached, most of the helicity is concentrated at large scales and the cascade is nonlocal. Helicity is transferred directly from the forced scales to the largest scales. At the same time, a smaller in amplitude direct cascade is observed from the largest scale to small scales.
\end{abstract}

Subject headings: magnetic fields - MHD

\section{INTRODUCTION}

The generation of magnetic fields in various astrophysical objects ranging from planets (e.g., the geodynamo; Glatzmaier \& Roberts 1996; Kono \& Roberts 2002) to stars (e.g., the solar dynamo; Dikpati \& Charbonneau 1999; Nandy \& Choudhuri 2002; Bushby \& Mason 2004) and spiral galaxies (e.g., the interstellar dynamo; Shukurov \& Dormy 2005) is mostly attributed to dynamo action due to motions of a conducting fluid (Moffatt 1978). Because of magnetic flux conservation in ideal magnetohydrodynamics (MHD), the stretching of magnetic field lines by a conducting flow amplifies the magnetic energy at small scales. To further explain how magnetic fields end up in scales much larger than the outer scales offluid motions, one of the theoretical arguments used is the inverse cascade of magnetic helicity in MHD turbulence. It is worth mentioning here that the presence of helicity in the flow, although helpful, is not required to generate largescale magnetic fields. In some circumstances, large-scale fields can be sustained solely by helicity fluctuations (Gilbert et al. 1988), by anisotropic flows (Nore et al. 1997), or by large-scale shear (Urpin 2002). However, magnetic helicity plays a fundamental role in the long-term evolution of stellar and galactic magnetic fields.

The solar case is of particular interest because theories on the generation of helical magnetic fields can be tested by a large number of observations. Direct measurements of magnetic helicity can be made in coronal mass ejections (CMEs). The S-shaped (and their mirror symmetric Z-shaped) structures observed in the Sun in soft X-rays (see Kurokawa 1987; Leka et al. 1996; Ishii et al. 1998), known as sigmoids, are attributed to the emergence of helical magnetic tubes (see Low 1994; Mandrini et al. 2004; Zhang \& Low 2005). It is the twist of the magnetic field lines that results in sigmoid observation (Low \& Berger 2003; Fan \& Gibson 2003, 2004; Gibson et al. 2004; Manchester et al. 2004). A correlation between twisting and the occurrence of CMEs has also been reported in Nindos et al. (2003) and Nindos \& Andrews (2004). The further evolution of CMEs can be followed from the Sun's photosphere to the interplanetary medium, where direct measurements of magnetic helicity can also be made (Smith 1999; Green et al.

\footnotetext{
1 National Center for Atmospheric Research, P.O. Box 3000, Boulder, CO 80307-3000.
}

2002; Démoulin et al. 2002; Mandrini et al. 2005). The observations made over the last two solar cycles have shown that S-shaped sigmoids appear preferentially in the southern hemisphere and Z-shaped sigmoids in the northern. This implies that structures in the northern and southern hemisphere have opposite signs of magnetic helicity. The origin of these helical magnetic fields with different signs of helicity in the northern and southern hemispheres is of fundamental interest in solar physics.

Since magnetic helicity is a conserved quantity, CMEs and magnetic clouds (MCs) in the interplanetary medium preserve the sign of the magnetic helicity of the active region where they were generated (Mandrini et al. 2005). The possible sources of magnetic helicity in stars such as the Sun are the $\Omega$ effect (associated with differential rotation in the base of the convective region), the $\alpha$ effect (associated with helical motions induced by the Coriolis force in the convective region), and surface photospheric motions (Berger \& Ruzmaikin 2000).

Recent studies showed that surface photospheric motions cannot provide enough magnetic helicity to explain the observations (Démoulin et al. 2002) or else give the wrong sign of magnetic helicity (Green et al. 2002). As a result, magnetic helicity is expected to be generated by subphotospheric motions, deep in the stellar convective region. Berger \& Ruzmaikin (2000) estimated the amount of magnetic helicity generated by the $\Omega$ effect and found that both the sign and the injection rate of magnetic helicity in each hemisphere is in good agreement with observations. On the other hand, estimations of the injection of magnetic helicity by helical motions of the flow (the $\alpha$ effect) are a matter of controversy. Kinetic helicity does not inject net helicity in the magnetic field but rather injects opposite signs of magnetic helicity at large and small scales (through kink and twist of the flux tubes). The evolution of the magnetic helicity at small scales is unclear. Direct numerical simulations of helical dynamo action (Brandenburg 2001) are done at moderate values of the magnetic Reynolds number, and as a result the small-scale magnetic helicity is dissipated relatively fast. The end result is a system dominated by the large-scale magnetic helicity. If the magnetic Reynolds number is much larger (as in the case in astrophysics) and both signs of magnetic helicity are assumed to cascade to large scales, the transfer of small-scale magnetic helicity to the dissipation length is expected to be inhibited. As a result, the 
injection of helicity by the $\alpha$ effect takes place in too large timescales (Berger \& Ruzmaikin 2000). However, the answer would be different if the fate of the small-scale magnetic helicity is different from the fate of the large-scale helicity.

In the galactic dynamo, the evolution of small-scale magnetic helicity also plays a fundamental role. The saturation level of the magnetic field, as well as the saturation timescale, in several models (Kleeorin et al. 2002, 2003) depends on assumptions on the diffusion of magnetic helicity by small-scale velocity fluctuations. The results we discuss here can help to enhance such models.

The generation and cascade of magnetic helicity has been investigated in early studies using mean field theory (Steenbeck et al. 1966; Krause \& Rädler 1980; Blackman \& Field 2002) and turbulent closure models (Frisch et al. 1975; Pouquet et al. 1976). It was shown within the framework of the approximations made that magnetic helicity cascades inversely from small scales to large scales. Direct numerical simulations (DNSs; Pouquet \& Patterson 1978; Meneguzzi et al. 1981; Kida et al. 1991; Brandenburg 2001; Maron \& Blackman 2002; Gómez \& Mininni 2004) have verified the inverse cascade of magnetic helicity including in the highly compressible case (Balsara \& Pouquet 1999) and have shown the generation of large-scale magnetic fields from small-scale helical force. A detailed examination of the cascading process was investigated in Brandenburg (2001), in which the rate of transfer of magnetic energy among different scales was measured from DNSs. The results showed evidence of nonlocal energy transfer of magnetic energy from the small scales to the large scales, also suggesting a nonlocal transfer of magnetic helicity. However, in threedimensional MHD turbulence the ideal invariant that can display an inverse cascade, sensu stricto, is the magnetic helicity, not the magnetic energy, and no direct attempt to measure its transfer in simulations has been done so far.

In this paper we focus on helical flows and revisit the problem of the inverse cascade of the magnetic helicity by analyzing two DNSs: one forced through the induction equation by an external electromotive force and one forced mechanically. Although our motivation is the origin of large-scale magnetic fields in astrophysical objects, the complexity of the problem forces us to study the magnetic field in much simpler triple periodic domains. In both simulations, the force was applied at small scales so that enough large scales were available for an inverse cascade to develop. Note that this election naturally limits the Reynolds numbers we can resolve, and as a result only moderate Reynolds numbers are considered in this work. Extending the formalism used by Dar et al. (2001), Debliquy et al. (2005), Alexakis et al. (2005), and Mininni et al. (2005) for the transfer of the magnetic and kinetic energy, we directly measured the transfer rate of magnetic helicity among different scales, in those both larger and smaller than the forcing scales.

The outline of the paper is as follows. In $\S 2$ we present a brief review of the equations and the definition of transfer functions needed to study this problem. In $\S 3$ we give the results from the magnetically forced simulation, and in $\S 4$ we give the results from the mechanically forced simulation. Finally, we discuss the implications of our results in $\S 5$, where we also give our conclusions.

\section{THEORY AND DEFINITIONS}

To a good approximation the equations that describe the dynamics of an incompressible conducting fluid coupled to a magnetic field are given by

$$
\begin{gathered}
\partial_{t} \boldsymbol{u}+\boldsymbol{u} \cdot \nabla \boldsymbol{u}=-\nabla P+\boldsymbol{b} \cdot \nabla \boldsymbol{b}+\nu \nabla^{2} \boldsymbol{u}+\boldsymbol{f}, \\
\partial_{t} \boldsymbol{b}=\nabla \times(\boldsymbol{u} \times \boldsymbol{b})+\eta \nabla^{2} \boldsymbol{b}+\nabla \times \boldsymbol{E},
\end{gathered}
$$

where $\boldsymbol{u}$ is the velocity field, $\boldsymbol{b}$ is the magnetic field, $\nu$ is the kinematic viscosity, $\eta$ is the magnetic diffusivity, $P$ is the total pressure, $\boldsymbol{f}$ is an external mechanic force, and $\boldsymbol{E}$ is an external electromotive force. The equations are written in the familiar Alfvénic dimensionless units. These equations are accompanied by the conditions $\nabla \cdot \boldsymbol{u}=0=\nabla \cdot \boldsymbol{b}$. This last condition allows us to write the magnetic field in terms of a vector potential $\boldsymbol{b}=\nabla \times \boldsymbol{a}$. Removing a curl from equation (2), the evolution equation for the vector potential reads

$$
\partial_{t} \boldsymbol{a}=\boldsymbol{u} \times \boldsymbol{b}+\eta \nabla^{2} \boldsymbol{a}-\nabla \phi+\boldsymbol{E},
$$

where the Coulomb gauge $(\nabla \cdot \boldsymbol{a}=0)$ is assumed and $\nabla \phi$ is determined by the solenoidal condition on $\boldsymbol{a}$. There are three quadratic invariants in the absence of dissipation and force: the total energy $E=\int\left(\boldsymbol{b}^{2}+\boldsymbol{u}^{2}\right) / 2 d x^{3}$, the cross helicity $H_{c}=\int \boldsymbol{b} \cdot \boldsymbol{u} d x^{3}$, and the magnetic helicity $H_{m}=\int \boldsymbol{b} \cdot \boldsymbol{a} / 2 d x^{3}$. (Note that a factor of $\frac{1}{2}$ has been introduced in the definition of magnetic helicity, following the notation of Pouquet et al. [1976].) To the best of our knowledge the magnetic helicity, which is the quantity under investigation in this paper, was first introduced as an invariant of the MHD equations by Woltjer (1958). It is proportional to the number of linkages of the magnetic field lines (Moffatt 1978), as reflected by its relation with topological quantities such as the Gauss linking number (Wright \& Berger 1989; Berger 1997). The conservation of magnetic helicity is related with the frozen-in theorem of Alfvén. Being magnetic field lines material, a link can only change through reconnection of field lines, and therefore breaking of the frozen-in condition (e.g., through dissipation) is needed.

As we stated in $\S 1$, we want to quantify the rate at which helicity is transferred among the different scales of the magnetic field. To define the magnetic field and vector potential at different scales, we introduce the shell-filtered magnetic field and vector potential components $\boldsymbol{b}_{K}(\boldsymbol{x})$ and $\boldsymbol{a}_{K}(\boldsymbol{x})$. Here $\boldsymbol{a}_{K}(\boldsymbol{x}), \boldsymbol{b}_{K}(\boldsymbol{x})$ are real vector functions of space $(\boldsymbol{x})$ and the subscript $K$ indicates that the field has been filtered to keep only the modes whose wavevectors are in the Fourier shell $[K, K+1)$ (hereafter called shell $K$ ):

$$
\boldsymbol{a}_{K}(\boldsymbol{x})=\sum_{\boldsymbol{k} \in K} \tilde{\boldsymbol{a}}(\boldsymbol{k}) e^{i \boldsymbol{k} \cdot \boldsymbol{x}} \quad \text { and } \quad \boldsymbol{b}_{K}(\boldsymbol{x})=\sum_{\boldsymbol{k} \in K} \tilde{\boldsymbol{b}}(\boldsymbol{k}) e^{i \boldsymbol{k} \cdot \boldsymbol{x}},
$$

where $\tilde{\boldsymbol{a}}(\boldsymbol{k})$ and $\tilde{\boldsymbol{b}}(\boldsymbol{k})$ are the Fourier transforms of $\boldsymbol{a}(\boldsymbol{x})$ and $\boldsymbol{b}(\boldsymbol{x})$, respectively, and the sum is taken over all wavenumbers whose moduli belong to shell $K$, i.e., $K \leq|\boldsymbol{k}|<K+1$. Clearly, the sum of all the $K$-components gives back the original field, $\boldsymbol{b}=\sum_{K} \boldsymbol{b}_{K}$, and the filtering operation commutes with the curl operator, $\nabla \times \boldsymbol{a}_{K}=\boldsymbol{b}_{K}$. Any two fields $\boldsymbol{a}_{K}, \boldsymbol{b}_{Q}$ with $K \neq Q$ are orthogonal, i.e., $\int \boldsymbol{a}_{K} \cdot \boldsymbol{b}_{Q} d x^{3}=0$, since the two shells contain no common wavenumbers. A similar decomposition has been used by Alexakis et al. (2005) and Mininni et al. (2005) to study the cascade of energy.

We are interested in the rate at which magnetic helicity at a given shell $Q$ is transferred into a different shell $K$. From the MHD equations, taking the dot product of equation (3) with $\boldsymbol{b}_{K} / 2$, taking the dot product of equation (2) with $\boldsymbol{a}_{K} / 2$, adding them, and integrating over space, we finally obtain the evolution of the magnetic helicity $H_{m}(K)=\frac{1}{2} \int \boldsymbol{a}_{K} \cdot \boldsymbol{b}_{K} d x^{3}$ in shell $K$ :

$$
\begin{aligned}
\partial_{t} H_{m}(K)= & \sum_{Q} \int \boldsymbol{b}_{K} \cdot\left(\boldsymbol{u} \times \boldsymbol{b}_{Q}\right) d \boldsymbol{x}^{3} \\
& -\eta \int \boldsymbol{b}_{K} \cdot \nabla \times \boldsymbol{b}_{K} d \boldsymbol{x}^{3}+\int \boldsymbol{b}_{K} \cdot \boldsymbol{E}_{K} d \boldsymbol{x}^{3} .
\end{aligned}
$$


The total magnetic helicity is given by the sum $H_{m}=\sum_{K} H_{m}(K)$. We can rewrite equation (5) in a more compact form,

$$
\partial_{t} H_{m}(K)=\sum_{Q} T_{h}(K, Q)-\eta D_{h}(K)+F_{h}(K)
$$

where we have introduced the transfer function $T_{h}(Q, K)$, the helicity injection $F_{h}(K)$, and the helicity dissipation $D_{h}(K)$ as defined below.

The dissipation of magnetic helicity in shell $K$ is given by

$$
D_{h}(K)=\int \boldsymbol{b}_{K} \cdot\left(\nabla \times \boldsymbol{b}_{K}\right) d \boldsymbol{x}^{3}
$$

Note, however, that unlike the energy dissipation this is not a positive definite quantity.

The injection rate of magnetic helicity in shell $K$ by the external electromotive force $\boldsymbol{E}$ is given by

$$
F_{h}(K)=\int \boldsymbol{b}_{K} \cdot \boldsymbol{E}_{K} d \boldsymbol{x}^{3}
$$

Note that the mechanical force $\boldsymbol{f}$ does not inject magnetic helicity in the system, as follows from equation (5). However, as discussed below, if the external mechanical force is helical, the velocity field can generate helical magnetic fields locally through the $T_{h}(Q, K)$ term.

The transfer rate of magnetic helicity at shell $Q$ into magnetic helicity at shell $K$ is defined as

$$
T_{h}(K, Q)=\int \boldsymbol{b}_{K} \cdot\left(\boldsymbol{u} \times \boldsymbol{b}_{Q}\right) d \boldsymbol{x}^{3},
$$

where $T_{h}(K, Q)$ expresses the transfer rate of positive helicity from shell $Q$ to shell $K$ or, equivalently, the transfer rate of negative helicity from shell $K$ into shell $Q$. Positive values of $T_{h}(K, Q)$ imply that positive helicity is transferred from shell $Q$ to shell $K$, while negative values imply the reverse transfer. The transfer term is a conservative term and does not generate or destroy total magnetic helicity. However, this term is responsible for the redistribution of magnetic helicity among different scales. This fact is expressed by the antisymmetric property of $T_{h}(Q, K)$ :

$$
T_{h}(K, Q)=-T_{h}(Q, K)
$$

We stress that helicity (unlike energy) is not a positive definite quantity, so care needs to be taken when we interpret results. We do not attempt here a separation of its different sign components (see, e.g., Waleffe [1992] and Chen et al. [2003a, 2003b] for the kinetic helicity in hydrodynamic turbulence). As an example, if in some shell $K$ the helicity is positive $H_{b}(K)>0$ with a positive rate of change $\partial_{t} H_{b}(K)>0$, then the magnetic field becomes more helical in that shell as the system evolves. If, however, the helicity is negative $H_{b}(K)<0$, then positive rate of change implies that the field becomes less helical in that shell. In the same spirit, if positive helicity is transferred from scales with negative helicity to scales with positive helicity, the field becomes more helical at both scales even if the total helicity at all scales remains constant. On the other hand, if positive helicity is transferred from scales with positive helicity to scales with negative helicity, the field becomes less helical at each scale, since the absolute value of magnetic helicity at each scale is decreased.

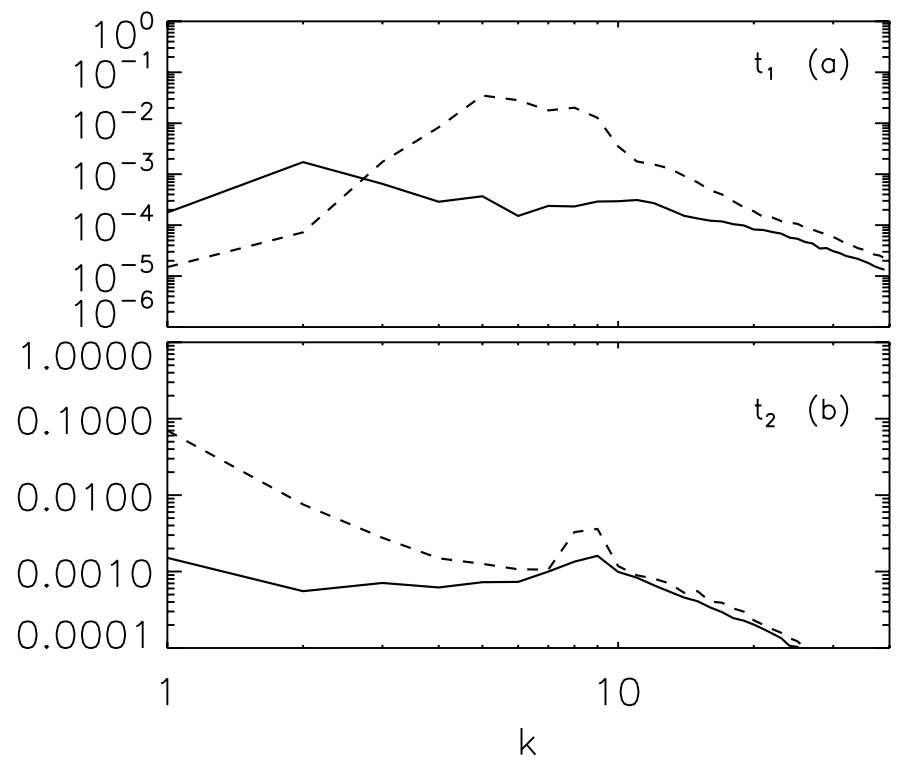

FIG. 1.-Kinetic (solid line) and magnetic (dashed line) energy spectra for the magnetically forced simulation for two different times: $(a) t_{1}$, before saturation is reached, and $(b) t_{2}$, close to saturation. Spectra are shown up to $k=40$; note, however, that the maximum wavenumber resolved in the code is $k_{\max } \approx 85$.

\section{MAGNETICALLY FORCED RUN}

We begin with the magnetically forced simulations. This case, although less physically motivated, provides a reference frame for the study of the inverse cascade of the magnetic helicity. Magnetic helicity is not a positive definite quantity, and as a result the interpretation of its transfer is complex. While in astrophysical dynamos magnetic energy is believed to be sustained by motions of a conducting fluid, the injection of magnetic energy and helicity directly in the induction equation allows us to have systems dominated by only one sign of magnetic helicity at all scales. As a result, the interpretation of the transfer is straightforward, and the results in this section can be used as a reference to validate the direction of the cascades in the more realistic simulations of $\S 4$.

In the numerical simulation, a helical $\mathrm{ABC}$ electromotive force $\boldsymbol{E}$ is used, while keeping the mechanical force equal to zero. The flow evolution is solved using a pseudospectral method with the two-thirds rule for dealiasing on a $N^{3}=256^{3}$ grid. No uniform magnetic fields are allowed in the periodic box, and therefore magnetic helicity conservation is satisfied in the ideal case (Berger 1997).

The viscosity and diffusivity are set to $\nu=\eta=5 \times 10^{-4}$. Only wavenumbers in shells 8 and 9 are forced. The phase of the external $\mathrm{ABC}$ electromotive force is changed randomly with a correlation time $\tau=1.25 \times 10^{-2}$, and the time step to satisfy the Courant-Friedrichs-Levy (CFL) condition is set to $\Delta t=2.5 \times$ $10^{-3}$. The integral length scale of the flow $L=2 \pi \int E(K) / K d k / E$ [where $E(K)$ is the kinetic energy in shell $K$ and $E$ is the total kinetic energy] in the steady state of the simulation is $L \approx 1.42$, and the large-scale turnover time $T=U / L$ (where $U$ is the rms velocity) is $T \approx 0.25$. Based on these numbers, the resulting kinetic Reynolds number $\operatorname{Re}_{e}$ and magnetic Reynolds number $\mathrm{Re}_{m}$ are $\mathrm{Re}_{e} \approx \mathrm{Re}_{m} \approx 700$. The simulation is well resolved, in the sense that the Kolmogorov kinetic $\left[k_{\nu}=\left(\epsilon / \nu^{3}\right)^{1 / 4}\right.$, where $\epsilon$ is the total energy injection rate $]$ and magnetic $\left[k_{\eta}=\left(\epsilon / \eta^{3}\right)^{1 / 4}\right]$ dissipation wavenumbers are smaller than the maximum resolved wavenumber $k_{\max } \approx N / 3$.

The magnetically forced case is easier to analyze because only one sign of helicity appears to dominate all scales. In Figure 1 we 
show the resulting spectra at two different times $t_{1}$ and $t_{2}$, up to wavenumber $k=40$. The former time is early (before the system comes close to saturation), and the latter time is when the flow is almost saturated. Note that the maximum wavenumber in the code is $k_{\max } \approx 85$ and that the dissipative range in the simulation extends to larger wavenumbers than what is shown in Figure 1 . However, the transfer function $T_{h}(K, Q)$ is only computed up to $K, Q=40$, a range that includes all scales larger (wavenumbers smaller) than the injection band, as well as the scales smaller than the injection band where a turbulent scaling can still be identified. We follow this convention in the following figures.

\subsection{Early Times}

The spectrum of magnetic helicity for $t=t_{1}$ is shown in Figure $2 a$ in log-log scale and is positive at all scales. At this stage, the magnetic helicity spectrum peaks at wavenumber $K \simeq 5$, a scale slightly larger than the scale where the system is forced. Figures $2 b-2 e$ show the transfer of magnetic helicity $T_{h}(K, Q)$ at different values of $Q$, normalized by the total magnetic helicity in that shell. The dotted lines in these panels indicate the shell where the force is applied, while the dashed lines indicate the mode that is examined.

Since the helicity is positive for all scales, we only need to interpret $T_{h}$ as a transfer of positive helicity. Figure $2 b$ shows the transfer $T_{h}(K, Q)$ for wavenumbers at the peak of the energy spectrum $(K=5)$. For smaller wavenumbers $(K<5)$ the transfer is positive, while it is negative for larger wavenumbers $(K>5)$. This picture indicates that the shell $K=5$ is giving/transferring helicity to its close neighbors on the left, while it receives helicity from its neighbors on the right.

Similar behavior is observed for the modes with wavenumbers between the peak of magnetic helicity in Fourier space and the forcing wavenumber (see Fig. $2 a$ ). The transfer of magnetic helicity for a value of $Q$ in this range $(Q=7)$ is shown in Figure $2 c$, suggesting the picture of a local inverse cascade. Indeed, the shell $Q=7$ gives most of its helicity to the shell $K \approx 6$ (positive peak), while it receives helicity from the shell $K \approx 8$ (negative peak).

The forced wavenumbers [the transfer $T_{h}(K, Q)$ for $Q=8$ is shown in Fig. $2 d$ ] are giving helicity to both smaller and larger scales, with a preference toward the larger scales (smaller wavenumbers). Finally, wavenumbers larger than the forced scale (Fig. 2e) have a different behavior. Unlike the large scales, the small scales $(K=20$ is displayed here) receive helicity from larger scales (but smaller than the forced scale) and give helicity to smaller scales. This suggests a local direct cascade of positive magnetic helicity. In addition, there is a nonlocal transfer of helicity to much larger scales $(K \simeq 5)$, probably associated with reconnection events.

\subsection{Late Times}

The picture of a local cascade of helicity is changed at later times, as the peak of the helicity spectrum moves to the largest possible scale $(K=1)$. The helicity spectrum and the transfer functions at this stage are shown in Figure 3. The helicity spectrum peaks strongly at $K=1$ (see Fig. $3 a$ ). As shown in Figure $3 b$, the largest scales are now receiving magnetic helicity directly from the forced scales, the remaining scales giving almost zero contribution. This behavior suggests a nonlocal inverse cascade. Intermediate scales between the largest available and the forced scales have also changed their behavior (Fig. $3 c$ ). These scales $(1<K<8)$ now are receiving positive helicity mostly
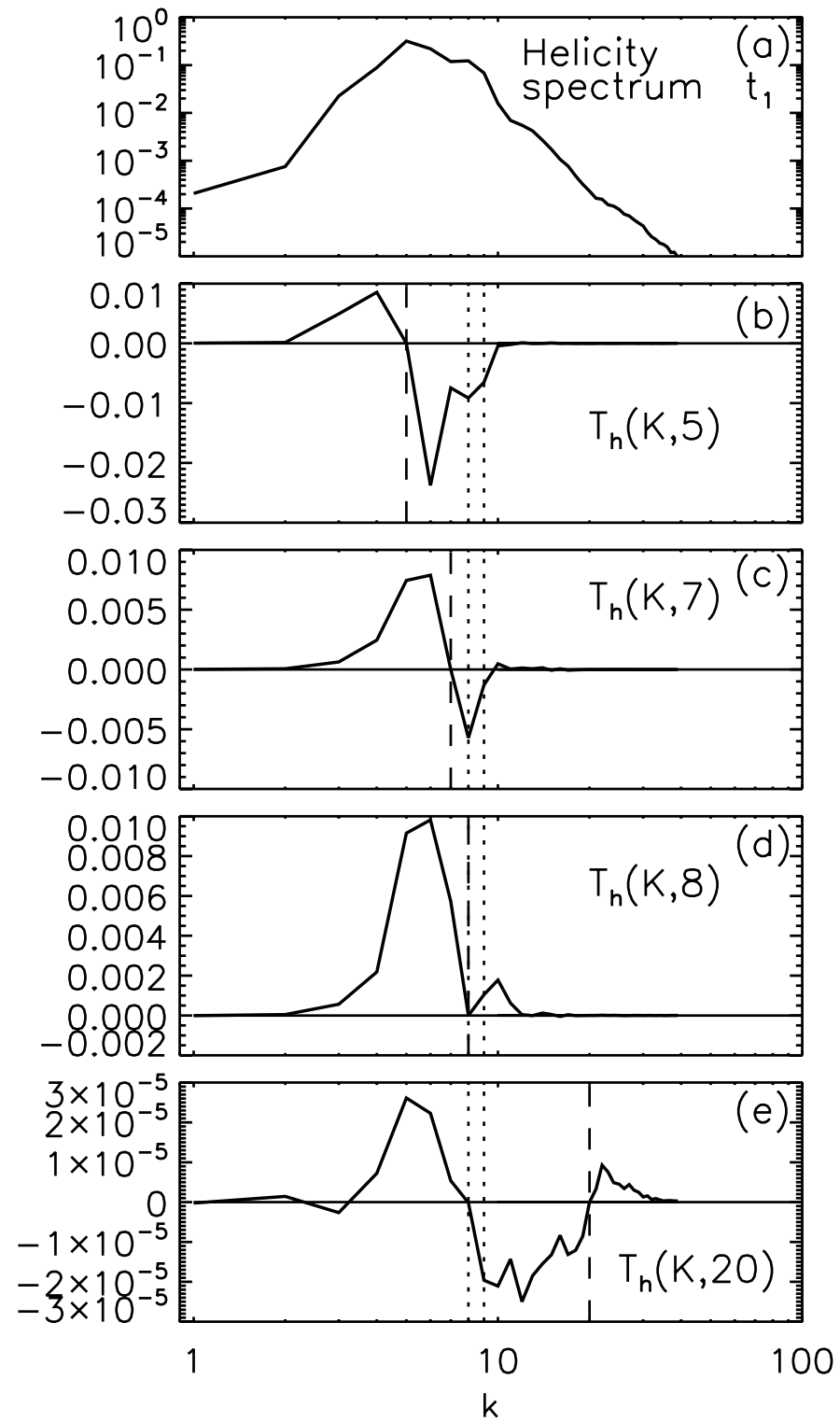

FIG. 2.- (a) Magnetic helicity spectrum and $(b-e)$ its transfer $T_{h}(K, Q)$ from shell $Q$ to shell $K$, normalized by the total magnetic helicity for the magnetically forced run at time $t_{1}$. Panels $(b-e)$ correspond to $Q=5,7,8$, and 20 , respectively. The dashed vertical line indicates the location of the examined value of $Q$, while the dotted lines give the width of the forcing band. Note that the transfer for $Q=20$ is significantly smaller.

from the largest modes and giving it to the smaller scales. This suggests a direct cascade of positive magnetic helicity in the range $1<K<8$. Therefore, it appears that once the magnetic helicity has reached the largest possible scale there may be some "reflection" at $K=1$, and helicity then cascades to smaller scales with the exception of the forced modes that continue feeding the magnetic helicity at the largest scale (see Fig. $3 d$ ). Scales smaller than the injection band transfer the positive magnetic helicity to smaller scales as at early times, with the exception of a nonlocal direct input to the largest scale.

The "reflection" of magnetic helicity in Fourier space when it reaches the largest scale in the box suggests that the late time evolution is strongly dependent on the boundary conditions. In our case, the periodic boundary conditions do not allow magnetic helicity to grow at even larger scales and forbid the system to eject magnetic helicity outside the box. Similar behavior has been observed in two-dimensional hydrodynamic turbulence, in 

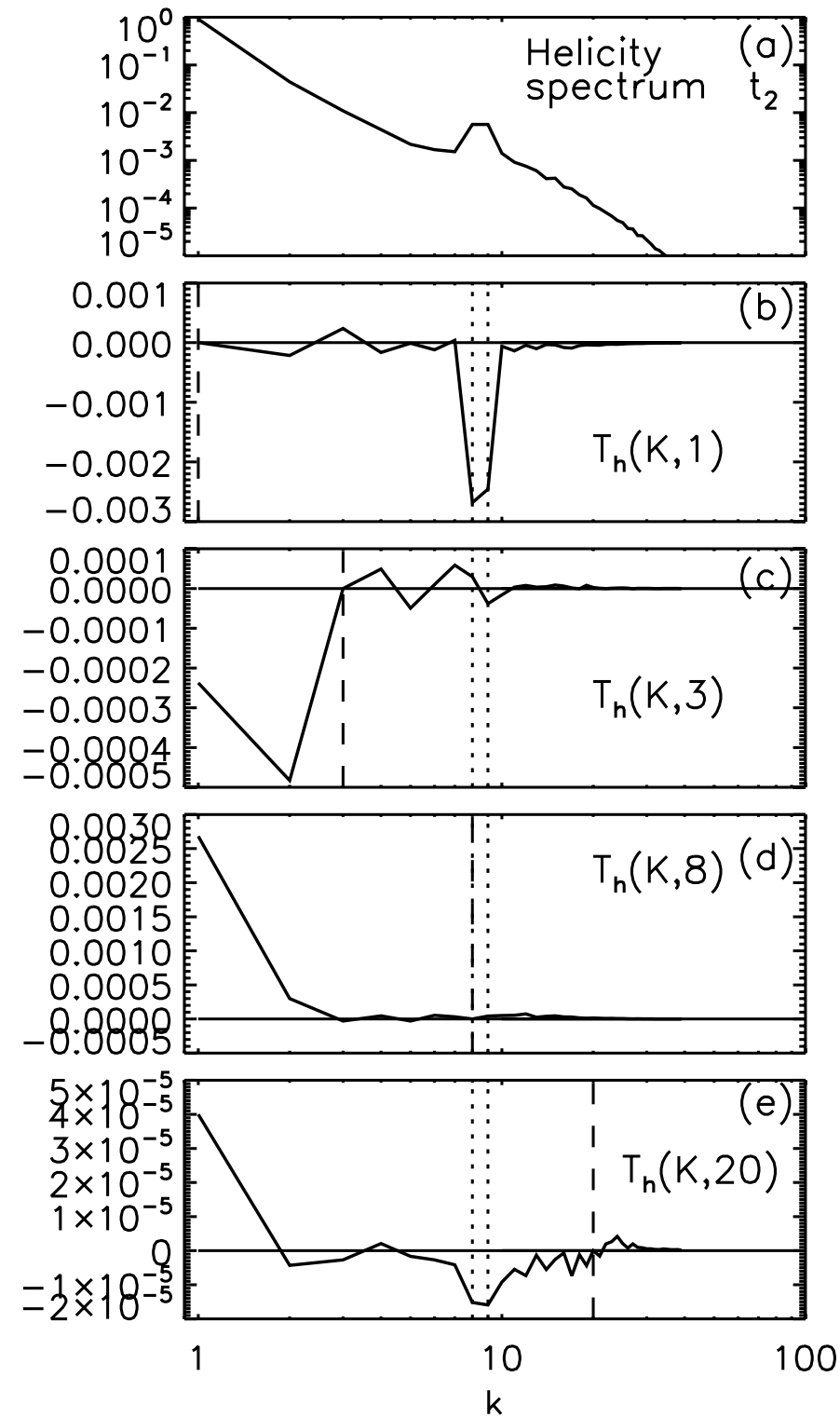

FIG. 3.- Same as Fig. 2, but at later times and with panels $(b-e)$ corresponding to $Q=1,3,8$, and 20, respectively. Note the different values on the vertical axis.

which the quantity that has an inverse cascade is the energy (Smith \& Yakhot 1994; Borue 1994; Boffetta et al. 2000). For this latter problem, evidence of nonlocal and irregular transfer of the inverse cascading invariant was also found in simulations (Danilov \& Gurarie 2001). We come back to this issue below.

\section{MECHANICALLY FORCED RUN}

We move next to the case in which the system is mechanically forced and the magnetic field is solely amplified and sustained against ohmic dissipation by dynamo action. This case is more relevant to most physical situations. In this case, we perform a numerical simulation using a grid of $N^{3}=256^{3}$ points under the following procedure. First, a hydrodynamic simulation was performed, mechanically forcing at wavenumber $K=10$, with an ABC flow to obtain a turbulent steady state. The kinetic helicity of the flow $H_{k}=\int \boldsymbol{u} \cdot \nabla \times \boldsymbol{u} / 2 d^{3} x$ in the steady state is positive and close to maximal. Unlike the previous section, here the phases of the $\mathrm{ABC}$ flow are kept constant as a function of time.
After reaching the hydrodynamic steady state, a random, nonhelical, and small magnetic field was introduced and the simulation was carried keeping the force fixed to observe the evolution of the system from the initial exponential amplification of the magnetic energy until the large-scale saturation. The kinematic viscosity and magnetic diffusivity were $\eta=\nu=2.5 \times$ $10^{-3}$. In the hydrodynamic steady state, the integral scale of the flow was $L \approx 0.6$ and the large-scale eddy turnover time $T \approx 0.6$. Based on these numbers, the mechanic and magnetic Reynolds numbers were $\operatorname{Re}_{e}=\operatorname{Re}_{m}=240$.

From equations (6) and (8), we note that a helical mechanical force cannot inject net magnetic helicity in the system. However, a flow with positive kinetic helicity in the forcing band generates equal amounts of magnetic helicity at large and small scales with opposite signs. This generation can be understood in a geometrical way from the stretch-twist-fold (STF) dynamo (Zel'dovich et al. 1983). As magnetic flux tubes at large scales are twisted in one direction (generating one sign of magnetic helicity), magnetic field lines at small scales are twisted in the opposite direction.

This generation of opposite signs of magnetic helicity at different scales is also a signature of the $\alpha$ effect (Seehafer 1996). In mean field theory (see, e.g., Krause \& Rädler 1980) the equation for the evolution of the mean magnetic helicity $\bar{H}_{m}$ is

$$
\partial_{t} \bar{H}_{m}=\int\left(\alpha \bar{B}^{2}-\beta \overline{\boldsymbol{B}} \cdot \nabla \times \overline{\boldsymbol{B}}\right) d^{3} x,
$$

where $\alpha \approx-\tau\langle\boldsymbol{v} \cdot \nabla \times \boldsymbol{v}\rangle$ is proportional to minus the kinetic helicity of the flow (here $v$ is the fluctuating velocity field and $\tau$ is a correlation time). The coefficient $\beta$ is a positive turbulent diffusivity. As a result, the $\alpha$ effect injects magnetic helicity with a sign opposite that of the kinetic helicity into the mean (large scale) magnetic field. As its counterpart, at small scales the fluctuating magnetic field receives magnetic helicity of the same sign as the kinetic helicity.

We investigate three different times. In the first case, the dynamo is still kinematic (i.e., the magnetic energy is smaller than the kinetic energy at all scales, and the effect of the Lorentz force on the velocity field can thus be neglected). In the second, kinetic and magnetic energies are of the same order, but the peak of the magnetic helicity is not yet at the largest scales. In this regime, scales smaller than the energy injection band have reached saturation, while the large-scale magnetic field keeps growing slowly. Finally, we investigate the saturated stage at which the magnetic helicity spectrum peaks at the largest attainable scale.

The energy spectra for these three cases are shown in Figure 4. As in $\S 3$, the maximum wavenumber resolved in the simulation was $k_{\max } \approx 85$, and at all times the Kolmogorov dissipation wavenumbers were smaller than $k_{\max }$. Since the transfer between different shells is only studied up to $K, Q=40$, all spectral quantities in the figures are shown up to this wavenumber.

\subsection{Kinematic Regime}

We begin with the kinematic regime. The magnetic helicity spectrum is shown in Figure $5 a$. Unlike the magnetically forced case, the magnetic helicity spectrum changes sign. For scales smaller than the forced scales, the magnetic helicity spectrum is positive, while at large scales the magnetic helicity is negative. The positive and negative peaks are close on either side of the forced band. The transfer of helicity $T_{h}(K, Q)$ for various shells $Q$ is shown in Figures $5 b-5 e$.

The large scales $(Q=5$ is shown in Fig. $5 b)$, where the negative peak of the magnetic helicity spectrum is located, receive 


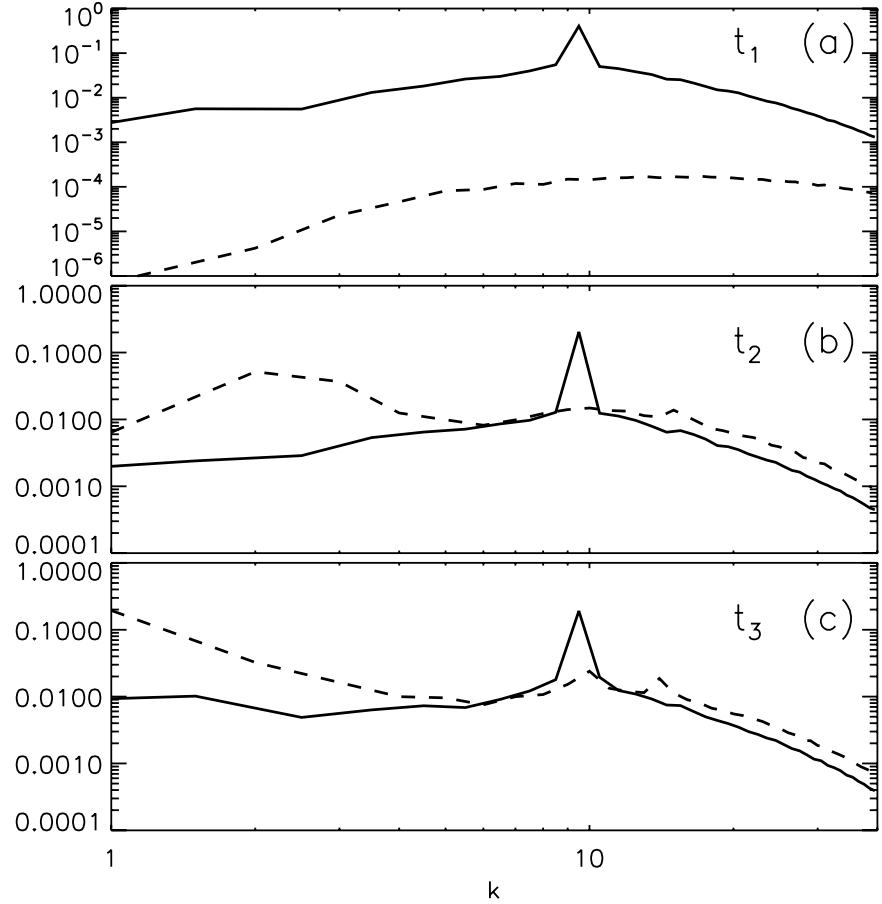

Fig. 4.- Same as Fig. 1, but for the mechanically forced simulation for three different times: $(a) t_{1}$, during the kinematic stage, $(b) t_{2}$, when small scales are saturated, and $(c) t_{3}$, when large scales are also close to saturation.

some negative helicity from smaller scales, but most of the transfer is from the forced scales $(K \approx 10)$. These scales also give (negative) helicity to larger scales (see Figs. $5 b$ and $5 c$ ). Note that because helicity is negative in the large scales, a positive value of $T_{h}(K, Q)$ means that shell $K$ receives negative magnetic helicity from shell $Q$, increasing the absolute value of the magnetic helicity in shell $K$, and the other way around if $T_{h}(K, Q)$ is negative.

The forced scale (see $Q=10$ in Fig. $5 d$ ), as described in the beginning of this section, is giving negative magnetic helicity to large scales and positive magnetic helicity to the small scales. This is the largest amplitude transfer and is the main source of "absolute" magnetic helicity. At scales smaller than the energy injection band (see Fig. 5e), like in the magnetically forced case, (positive) magnetic helicity appears to cascade to smaller scales, where it is finally dissipated.

\subsection{Small-Scale Saturated Regime}

As the amplitude of the magnetic field is increased by dynamo action, the growth of magnetic energy at scales smaller than the forcing band saturates. Meanwhile, the negative peak of the magnetic helicity moves to larger scales (see Fig. $6 a$ ). The large scales in the system $(K>10)$ receive (negative) magnetic helicity both locally from slightly smaller scales and nonlocally from the forced scales and give negative magnetic helicity to slightly larger scales if available (see Figs. $6 b-6 c$ ). The forced scale (corresponding to $Q=10$; see Fig. $6 d$ ) gives most of the negative magnetic helicity to the shell where the magnetic helicity spectrum peaks $(K \approx 2)$. At the same time, the forced shell gives positive magnetic helicity to slightly smaller scales. Finally, the small scales (Fig. 6e) cascade the positive magnetic helicity to even smaller scales, where it is dissipated. In addition, a considerable amount of magnetic helicity is destroyed by transferring positive helicity from the small scales (where the magnetic helicity is mostly positive) directly into the large scales (where helicity is negative; see the positive peak at $K \approx 2$ in Fig. $6 e$ ), as a
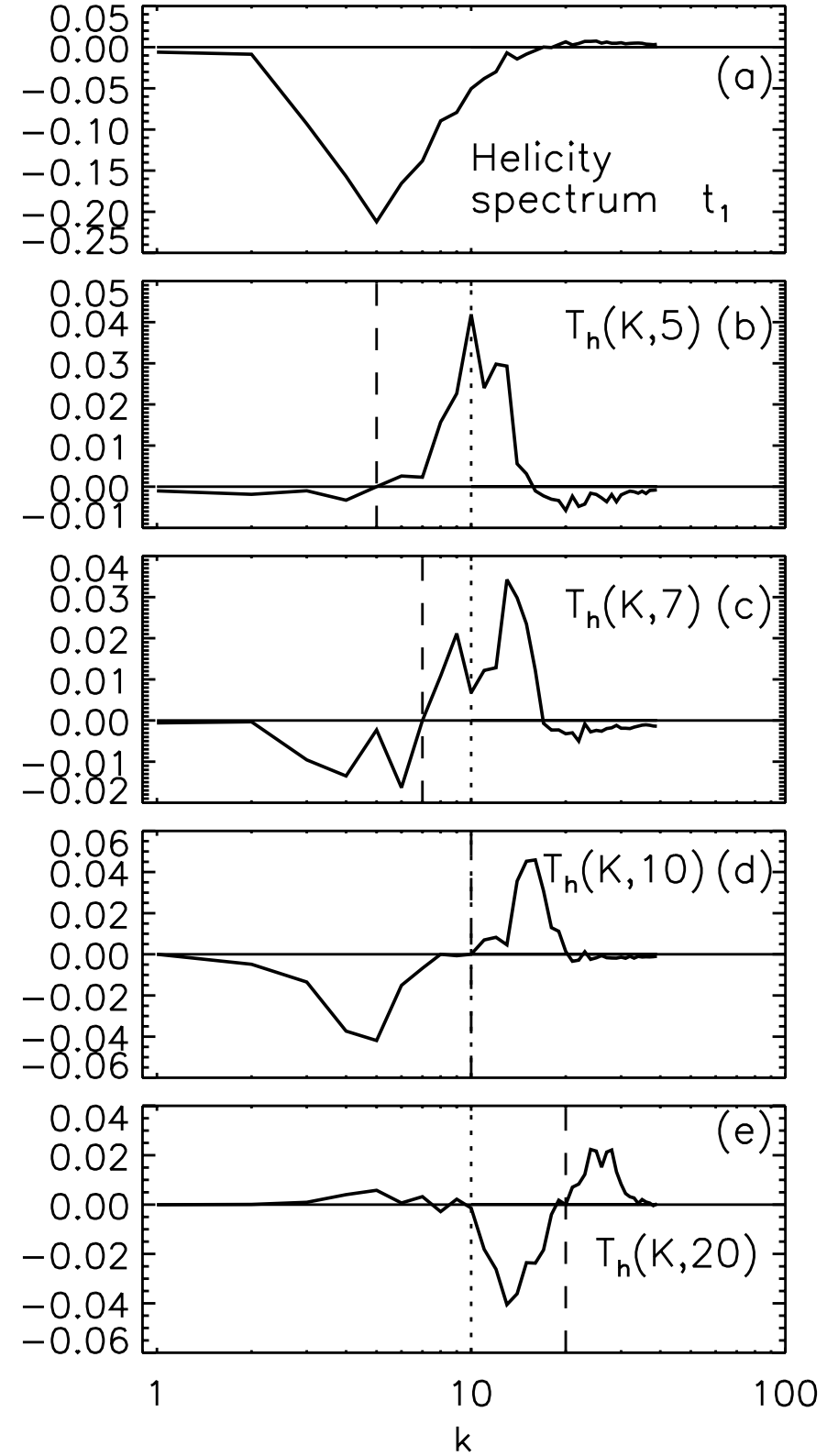

FIG. 5.- $(a)$ Spectrum and $(b-e)$ transfer of magnetic helicity $T_{h}(K, Q)$ normalized by the total magnetic helicity at shell $Q$ for the mechanically forced run in the kinematic regime. Panels $(b-e)$ correspond to $Q=5,7,10$, and 20 , respectively. The dashed vertical line indicates the location of the examined value of $Q$, while the dotted line indicates the shell where the system was forced.

result decreasing the absolute value of magnetic helicity in both scales. We believe this behavior may be related to reconnection events.

\subsection{Saturated Regime}

When the system is close to saturation at all scales, the helicity spectrum peaks at the largest available scale $(K=1$; see Fig. 7a). At this stage the large scales receive magnetic helicity directly from the forced scales by a nonlocal process (see Figs. $7 b$ and $7 d$ ). Such behavior has also been observed for the transfer of magnetic energy in helical dynamo runs by Brandenburg (2001).

In the intermediate scales, between the largest available scale in the box and the forced scale (see Fig. 7c), there seems to be a direct cascade of helicity from the large scales to smaller scales. 

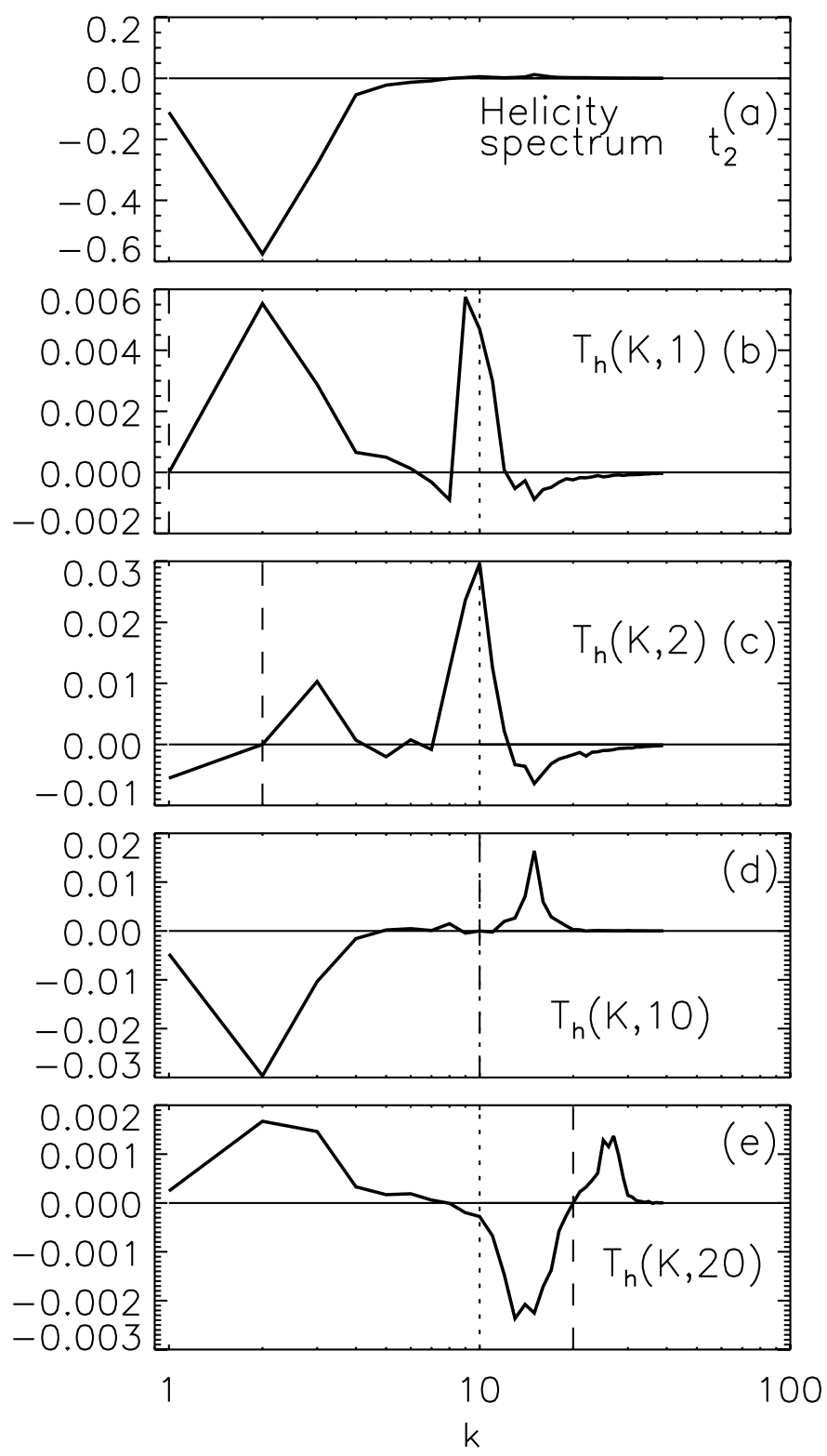

FIG. 6.- Same as Fig. 5, but when the small scales in the system are saturated and with panels $(b-e)$ corresponding to $Q=1,2,10$, and 20 , respectively.

This direct cascade of helicity at large scales is similar to the "reflection" of magnetic helicity at $K=1$ observed in the magnetically forced run (see $\S 3$ ) and is also expected to be dependent on the boundary conditions.

The forcing band keeps injecting magnetic helicity of opposite signs at large and small scales (Fig. $7 d$ ), but while positive magnetic helicity is injected at wavenumbers slightly larger than the forcing shell $Q=10$, most of the negative magnetic helicity is injected nonlocally into the shell $K=1$. Scales smaller than the forced scale (see Fig. 7e) cascade the positive magnetic helicity to smaller scales, where it is dissipated. Again, there is a nonlocal transfer of positive helicity from the small scales to the largest scale (see the positive peak at $K=1$ ) leading to the decrease in the absolute value of the magnetic helicity in both scales. Note that as the result of the inverse cascade of one sign of magnetic helicity at large scales and the direct cascade of magnetic helicity of the opposite sign at small scales, the system is finally dominated by magnetic helicity of sign opposite that of the kinetic helicity injected by the mechanical force. This
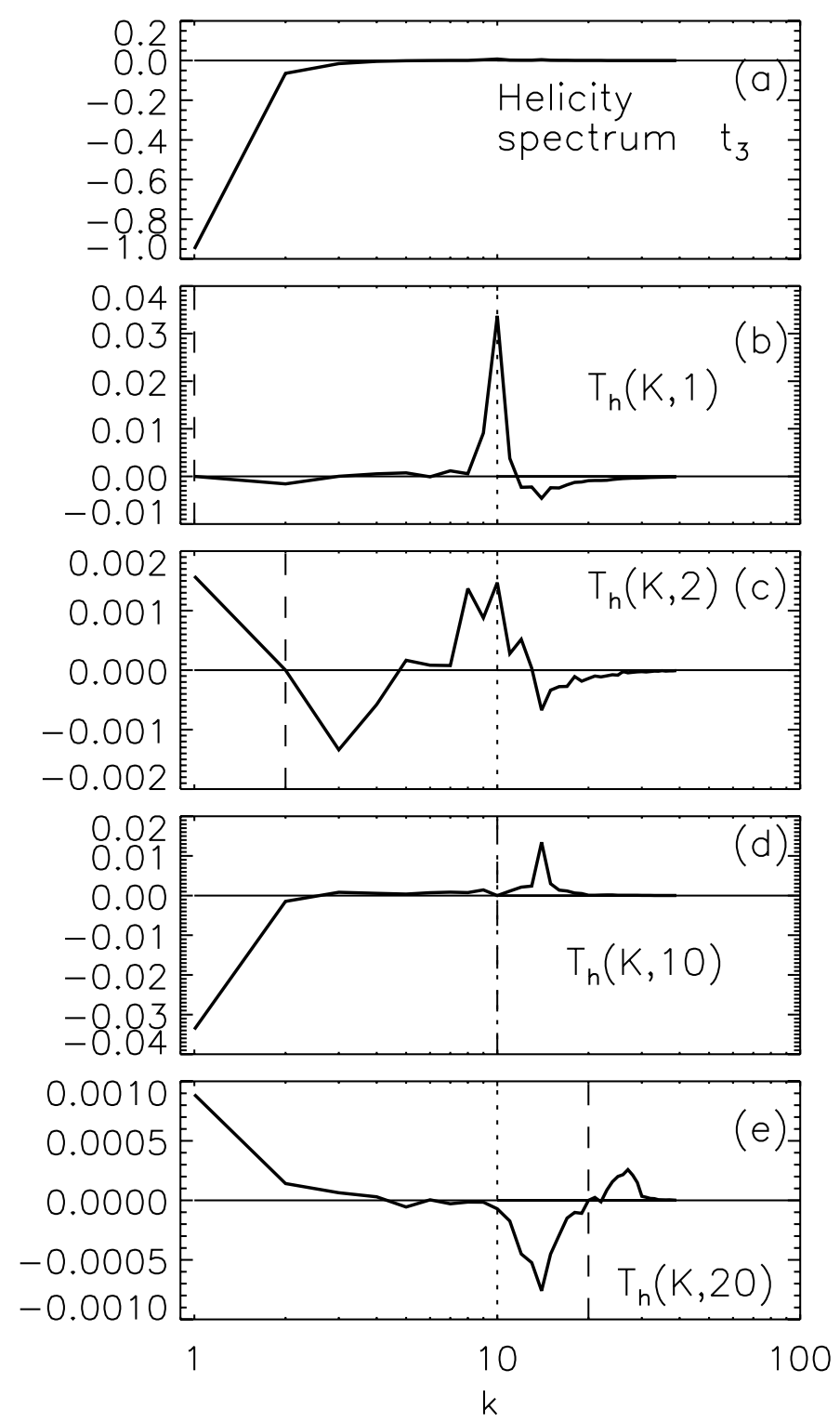

FIG. 7.-Same as Fig. 5, but close to saturation at large scales and with panels $(b-e)$ corresponding to different values of $Q=1,2,10$, and 20 , respectively.

has been observed by Brandenburg (2001) and in closures by Pouquet et al. (1976).

\section{DISCUSSION AND CONCLUSIONS}

The results presented above stemming from two numerical simulations have some important implications that need to be discussed. We start by giving a brief summary of what is observed in the simulations. At the early stages of the evolution of the magnetic field, in both runs, the peak of the magnetic helicity spectrum appears to be close to the forcing scales although in scales that are slightly larger. Magnetic helicity inversely cascades in the large scales, both locally by transfer of helicity from the closest neighbor shells and nonlocally by direct transfer from the forced shells. As the systems evolve, the inverse cascade of magnetic helicity leads the magnetic helicity spectrum to peak at the largest available scale in the domain. At this stage, the direct input coming from the nonlocal transfer of magnetic helicity from the forced scales to the largest attainable scales becomes dominant. At the same time, the local transfer of helicity at intermediate scales changes direction, and magnetic helicity cascades locally to small 
scales. This direct cascade between the largest scale in the box and the forcing band can be expected to be sensitive to the boundary conditions and is a nonuniversal feature common to other systems displaying inverse cascade. Similar behavior has been observed in two-dimensional hydrodynamic turbulence (Smith \& Yakhot 1994; Borue 1994; Boffetta et al. 2000; Danilov \& Gurarie 2001). However, we note that the nonlocal transfer from the forced scales to the large scales is much greater in amplitude than the local direct cascade. This behavior raises the interesting question of which process, the local or nonlocal cascade, is dominant in open systems such as stars or galaxies where no largest available scale can be clearly defined or where stellar or galactic winds can eject part of the magnetic helicity out of the system.

The small scales behave differently. Unlike the large scales, in the small scales there is a noticeable direct cascade of magnetic helicity to the dissipation scale. This implies that in the limit of infinite Reynolds number in a helically forced flow, at the saturated stage there is still going to be a finite global magnetic helicity, since one sign of magnetic helicity at scales larger than the forced scales will cascade inversely, while the opposite sign of magnetic helicity at small scales will cascade to smaller and smaller scales until it is dissipated. This is important when comparing the results from numerical simulations with astrophysical objects, since the typical Reynolds numbers in the latter case are much larger.

It can be argued that this direct cascade of small-scale magnetic helicity is counterintuitive (in the sense of self-similarity), since at a given scale the flow "does not know" if it is at scales smaller or larger than the forcing scale. Therefore, the flow could have been expected to see the same direction of cascade at all scales. This kind of argument, however, assumes that each scale is completely independent, but this is not the case for MHD. Magnetic helicity at scales larger than the integral scale of a helical flow is generated by the twisting and folding offlux tubes, forcing them to interpenetrate (Zel'dovich et al. 1983). At the same time, at small scales the twisting causes the magnetic field lines to spiral around each other, generating small-scale magnetic helicity of the sign opposite that at the large scale. Any further stretching of the flux tube will cause the small-scale magnetic helicity (i.e., the twisting of the field lines around the flux tube) to cascade to even smaller scales, even if the largescale helicity is cascading to larger scales. Furthermore, recon- nection at small scales changes the topology and the linkage of the field lines at large scales, which explains the nonlocal transfer of helicity from small scales to large scales, "destroying" in that way the large-scale helicity as it is observed in Figures 6 and $7 e$.

Therefore, while we confirm the well-known inverse cascade of magnetic helicity in a dynamo (Pouquet et al. 1976) at scales larger than the energy injection scale, we observe a novel direct cascade of magnetic helicity of the opposite sign for scales smaller than the forcing scale. As a result of the direct cascade of magnetic helicity at small scales, estimations of the diffusion time of the small-scale magnetic helicity (Berger \& Ruzmaikin 2000) should be revisited. Assuming there is an inverse cascade at all scales, the small-scale magnetic helicity dissipates in long times (of the order of the large-scale diffusion time $L^{2} / \eta$, where $L$ is a characteristic large scale of the system). However, if the small-scale helicity cascades to smaller scales, the dissipation timescale is of the same order as the dissipation timescale of the magnetic energy, a much faster process. As a result, the $\alpha$ effect can be expected to inject magnetic helicity even for large magnetic Reynolds numbers as is the case in astrophysics.

We also note that the direct cascade of magnetic helicity at small scales also is of relevance for several galactic dynamo models, in which diffusion of magnetic helicity by the turbulent fluctuations is required (Kleeorin et al. 2002, 2003).

We conclude by remarking that the overall picture of the cascade of magnetic helicity appears to be more complicated than that of the energy and crucially depends on the scale and the domain size. Simple assumptions carried over from hydrodynamic turbulence phenomenology do not seem to apply here. Boundary effects and flux transfer are other issues that should be investigated. Future numerical simulations, experiments, and refined theoretical arguments are needed in order to further illuminate the understanding of MHD turbulence and improve the modeling of turbulent flows.

Computer time was provided by NCAR. The NSF grant CMG-0327888 at NCAR supported this work in part and is gratefully acknowledged. The authors are grateful for valuable comments from B. C. Low and C. Mandrini.
Alexakis, A., Mininni, P. D., \& Pouquet, A. 2005, Phys. Rev. E, 72, 046301

Balsara, D., \& Pouquet, A. 1999, Phys. Plasmas, 6, 89

Berger, M. A. 1997, J. Geophys. Res., 102, 2637

Berger, M. A., \& Ruzmaikin, A. 2000, J. Geophys. Res., 105, 10481

Blackman, E. G., \& Field, G. B. 2002, Phys. Rev. Lett., 89, 265007

Boffetta, G., Celani, A., \& Vergassola, M. 2000, Phys. Rev. E, 61, 29

Borue, V. 1994, Phys. Rev. Lett., 72, 1475

Brandenburg, A. 2001, ApJ, 550, 824

Bushby, P., \& Mason, J. 2004, Astron. Geophys., 45, 7

Chen, Q., Chen, S., \& Eyink, G. L. 2003a, Phys. Fluids, 15, 361

Chen, Q., Chen, S., Eyink, G. L., \& Holm, D. D. 2003b, Phys. Rev. Lett., 90, 214503

Danilov, S., \& Gurarie, D. 2001, Phys. Rev. E, 63, 061208

Dar, G., Verma, M. K., \& Eswaran, V. 2001, Physica D, 157, 207

Debliquy, O., Verma, M. K., \& Carati, D. 2005, Phys. Plasmas, 12, 042309

Démoulin, P., Mandrini, C. H., van Driel-Gesztelyi, L., Thompson, B. J.,

Plunkett, S., Kovári, Zs., Aulanier, G., \& Young, A. 2002, A\&A, 382, 650

Dikpati, M., \& Charbonneau, P. 1999, ApJ, 518, 508

Fan, Y., \& Gibson, S. E. 2003, ApJ, 589, L105 2004, ApJ, 609, 1123

Frisch, U., Pouquet, A., Léorat, J., \& Mazure, A. 1975, J. Fluid Mech., 68, 769

Gibson, S. E., Fan, Y., Mandrini, C., Fisher, G., \& Demoulin, P. 2004, ApJ, 617, 600
REFERENCES

Gilbert, A. D., Frisch, U., \& Pouquet, A. 1988, Geophys. Astrophys. Fluid Mech., 42, 151

Glatzmaier, G. A., \& Roberts, P. H. 1996, Science, 274, 1887

Gómez, D. O., \& Mininni, P. D. 2004, Nonlinear Processes Geophys., 11, 619 Green, L. M., López fuentes, M. C., Mandrini, C. H., Démoulin, P., Van DrielGesztelyi, L., \& Culhane, J. L. 2002, Sol. Phys., 208, 43

Ishii, T. T., Kurokawa, H., \& Takeuchi, T. T. 1998, ApJ, 499, 898

Kida, S., Yanase, S., \& Mizushima, J. 1991, Phys. Fluids A, 3, 457

Kleeorin, N., Moss, D., Rogachevskii, I., \& Sokoloff, D. 2002, A\&A, 387, 453 2003, A\&A, 400, 9

Kono, M., \& Roberts, P. H. 2002, Rev. Geophys., 40, 4

Krause, F., \& Rädler, K.-H. 1980, Mean-Field Magnetohydrodynamics and Dynamo Theory (Oxford: Pergamon)

Kurokawa, H. 1987, Sol. Phys., 113, 259

Leka, K. D., Canfield, R. C., McClymont, A. N., \& van Driel-Gesztelyi, L. 1996, ApJ, 462, 547

Low, B. C. 1994, Phys. Plasmas, 1, 1684

Low, B. C., \& Berger, M. A. 2003, ApJ, 589, 644

Manchester, W., Gombosi, T., DeZeeuw, D., \& Fan, Y. 2004, ApJ, 610, 588

Mandrini, C. H., Démoulin, P., Van Driel-Gesztelyi, L., Green, L. M., \& López Fuentes, M. C. 2004, Ap\&SS, 290, 319

Mandrini, C. H., Pohjolainen, S., Dasso, S., Green, L. M., Démoulin, P., van

Driel-Gesztelyi, L., Copperwheat, C., \& Foley, C. 2005, A\&A, 434, 725

Maron, J., \& Blackman, E. G. 2002, ApJ, 566, L41 
Meneguzzi, M., Frisch, U., \& Pouquet, A. 1981, Phys. Rev. Lett., 47, 1060 Mininni, P. D., Alexakis, A., \& Pouquet, A. 2005, Phys. Rev. E, 72, 046302

Moffatt, H. K. 1978, Magnetic Field Generation in Electrically Conducting Fluids (Cambridge: Cambridge Univ. Press)

Nandy, D., \& Choudhuri, A. R. 2002, Science, 296, 1671

Nindos, A., \& Andrews, M. D. 2004, ApJ, 616, L175

Nindos, A., Zhang, J., \& Zhang, H. 2003, ApJ, 594, 1033

Nore, C., Brachet, M. E., Politano, H., \& Pouquet, A. 1997, Phys. Plasmas, 4, 1

Pouquet, A., Frisch, U., \& Léorat, J. 1976, J. Fluid Mech., 77, 321

Pouquet, A., \& Patterson, G. S. 1978, J. Fluid Mech., 85, 305

Seehafer, N. 1996, Phys. Rev. E, 53, 1283

Shukurov, A., \& Dormy, E. 2005, Introduction to Galactic Dynamos (Dordrecht: Kluwer)
Smith, C. W. 1999, in Magnetic Helicity in Space and Laboratory Plasmas, ed. M. R. Brown, R. C. Canfield, \& A. A. Pevtsov (Geophys. Monogr. 111; Washington: AGU), 239

Smith, L. M., \& Yakhot, V. 1994, J. Fluid Mech., 274, 115

Steenbeck, M., Krause, F., \& Rädler, K.-H. 1966, Z. Naturforsch., 21, 369

Urpin, V. 2002, Phys. Rev. E, 65, 026301

Waleffe, F. 1992, Phys. Fluids A, 4, 350

Woltjer, L. 1958, Proc. Natl. Acad. Sci., 44, 489

Wright, A. N., \& Berger, M. A. 1989, J. Geophys. Res., 94, 1295

Zel'dovich, Ia. B., Ruzmaikin, A. A., \& Sokoloff, D. D. 1983, Magnetic Fields in Astrophysics (New York: Gordon \& Breach)

Zhang, M., \& Low, B. C. 2005, ARA\&A, 43, 103 